\title{
From Betrayal to the Bottle: Investigating Possible Pathways From Trauma to Problematic Substance Use
}

\author{
Brianna C. Delker and Jennifer J. Freyd \\ Department of Psychology, University of Oregon, Eugene, Oregon, USA
}

\begin{abstract}
Research in both community and clinical settings has found that exposure to cumulative interpersonal trauma predicts substance use problems. Less is known about betrayal as a dimension of trauma exposure that predicts substance use, and about the behavioral and psychological pathways that explain the relation between trauma and substance use. In a sample of 362 young adults, this study evaluated three intervening pathways between betrayal trauma exposure prior to age 18 years and problematic substance use: (a) substance use to cope with negative affect, (b) difficulty discerning and/or heeding risk, and (c) self-destructiveness. In addition, exposure to trauma low in betrayal (e.g., earthquake) was included in the model. Bootstrap tests of indirect effects revealed that betrayal trauma prior to age 18 years was associated with problematic substance use via posttraumatic stress and two intervening pathways: difficulty discerning/heeding risk $(\beta=.07, p<.001)$, and self-destructiveness $(\beta=.12, p<.001)$. Exposure to lower betrayal trauma was not associated with posttraumatic stress or problematic substance use. Results contribute to a trauma-informed understanding of substance use that persists despite potentially harmful consequences.
\end{abstract}

Clinicians and researchers have long noted a striking cooccurrence between trauma exposure and problematic substance use, with definitions of the latter construct ranging from episodic binge drinking to chronic or compulsive substance use patterns that meet diagnostic criteria for a substance use disorder (Brady, Back, \& Coffey, 2004; Chilcoat \& Menard, 2003). Increasingly, research has identified exposure to cumulative interpersonal (as compared to noninterpersonal) trauma as a risk for substance use problems among treatmentseeking and community adults (Briere, Hodges, \& Godbout, 2010). Childhood physical and sexual abuse predict elevated risk for developing a substance use disorder in young adulthood (Afifi, Henriksen, Asmundson, \& Sareen, 2012), even after accounting for genetic and familial environmental risk (Duncan et al., 2008). Understanding why problematic substance use can develop from early interpersonal trauma is crucial for identifying and ameliorating risk for problem onset, particularly in young adulthood (ages 18-25), when rates of co-occurring substance use disorders and mental illness are higher than rates in any other age group (Substance Abuse and Mental Health Services Administration, 2012). An emerging explanation for increased substance use risk following early interpersonal trauma posits that substance use reflects

Correspondence concerning this article should be addressed to Brianna C. Delker, Department of Psychology, University of Oregon, 1227 University of Oregon, Eugene, OR 97403. E-mail: bcdelker@uoregon.edu

Copyright (c) 2014 International Society for Traumatic Stress Studies. View this article online at wileyonlinelibrary.com

DOI: $10.1002 /$ jts.21959 dysfunctional avoidance of posttraumatic negative affect states (Briere et al., 2010). This study seeks to affirm and extend the dysfunctional avoidance explanation by positing two additional betrayal trauma theory-driven pathways from early interpersonal trauma to problematic substance use: self-destructiveness and difficulty discerning and/or heeding risk to self or others.

Betrayal trauma theory suggests that when an individual depends on a perpetrator for material or emotional support, as in the case of a child who depends on an abusive caregiver, it may be adaptive to remain fully or partially unaware of the abuse (Freyd, DePrince, \& Gleaves, 2007). Less awareness of or less ability to recall aspects of ongoing, potentially traumatic betrayals can help the individual preserve a relationship important for survival. Over time, compromised awareness of betrayal may contribute to ongoing difficulty detecting risk in the social environment. For example, DePrince (2005) found that individuals with self-reported histories of betrayal trauma revictimization made significantly more errors when reasoning about social contract violations than did individuals with no revictimization experiences. Difficulty detecting social risk may in turn predict revictimization in adult relationships, including intimate relationships. For example, in a prospective study with college women, Messman-Moore and Brown (2006) found that delayed response to danger cues in a hypothetical social situation with a male acquaintance was associated with increased risk for rape revictimization over an 8-month follow-up period. The present study expands the concept of risk detection within intimate or dating contexts to include risk detection and evaluation as it relates to substance use. 
Survivors of betrayal trauma who develop problematic substance use behaviors may have difficulty discerning risk, or they may discern risk but disregard it. Reasons for disregarding acknowledged risk could range from a lack of concern for personal consequences that parallels the perpetrator's apparent lack of concern for the victim, to a sense of a foreshortened future that gives license to taking risks that threaten life or limb. For example, a qualitative study of adolescents found that some survivors of chronic physical and/or sexual abuse reported using alcohol because they began "to stop caring about what happened to them or what people thought about their behavior" (Rothman, Bernstein, \& Strunin, 2010). In this study, we hypothesize that problematic substance use-such as use characterized by a loss of control, a lack of concern for consequences, or physical danger-may reflect risk discernment and evaluation difficulties that emerge from a history of betrayal. Until further research distinguishes difficulty discerning risk from difficulty heeding it, we refer to this construct globally as difficulty regarding risk.

The second proposed pathway from early life betrayal trauma to problematic substance use is self-destructive ideation or behavior, including self-harm and suicidality, two known responses to interpersonal betrayal (Bornovalova, Tull, Gratz, Levy, \& Lejuez, 2011; Edwards, Freyd, Dube, Anda, \& Felitti, 2012). Victims of ongoing abuse may have experienced both physical threat to life or limb, and threat to the integrity of the social self, a violation of norms and expectations about the social world (Budden, 2009). One adaptation to the crumpling of safety and a sense of agency in the world associated with betrayal is to attribute betrayal not to the perpetrator's corruption of social norms and values, but to the victim's own supposedly corrupted self that somehow invited or justified the harm (Herman, 1997). The self-blame (Ullman, Filipas, Townsend, \& Starzynski, 2007) and shame (Platt \& Freyd, 2012) inherent in this adaptation to betrayal confer their own rationale for selfharm and suicidality. For survivors of early betrayal trauma, self-harm and suicidality may index a more or less intentional self-destructiveness.

The third proposed pathway from betrayal trauma to problematic substance use evaluated in this study is the use of substances to cope with posttraumatic negative affect states. In keeping with the constructs of dysfunctional avoidance (Briere et al., 2010), tension reduction (Greeley \& Oei, 1999), and self-medication (Khantzian, 1997), we propose that substance use may be one means of coping with or dulling awareness of trauma-related psychological distress. In support of the coping explanation, several studies with adolescent or adult participants diagnosed with substance use disorders have found that coping with unpleasant emotions is a more frequent trigger for substance use among those with (vs. without) co-occurring posttraumatic stress disorder (Staiger, Melville, Hides, Kambouropoulos, \& Lubman, 2009; Waldrop, Back, Verduin, \& Brady, 2007). In another study, coping motives partially mediated the association between posttraumatic stress and heavy drinking (Kaysen et al., 2007). In this study, we propose that use of substances to cope may be associated with problematic patterns of use, such as loss of control over use or using more of a substance than was intended.

The aim of this study was to identify psychological and behavioral pathways from early life betrayal trauma to problematic substance use in young adulthood, using a structural equation modeling approach (see Figure 1). We predicted that higher betrayal trauma prior to age 18 would be associated with posttraumatic stress, which in turn would be associated with (a) substance use to cope with negative affect, (b) difficulty regarding risk, and (c) self-destructiveness. Posttraumatic stress was included as a proposed link between betrayal and the three intervening pathways due to research that has identified associations between posttraumatic stress and coping motives (Peters, Khondkaryan, \& Sullivan, 2012), risk-taking behavior (Strom et al., 2012), self-harm (Weierich \& Nock, 2008), and suicidality (Panagioti, Gooding, \& Tarrier, 2012). In this study, we further hypothesized that the three intervening pathways would be associated with problematic substance use. Problematic substance use was modeled as a latent construct indexed by alcohol use quantity and frequency, harmful alcohol or drug use patterns, and loss of control over alcohol or drug use (Glantz, 2010). Finally, we examined lower betrayal trauma experiences as a control variable in the analysis.

\section{Method}

\section{Participants and Procedure}

Participants were drawn from 396 college students at a large, public university in the northwestern United States. The University of Oregon's Research Compliance Services organization approved the study protocol. Participants were recruited via an online research management system and received course credit for participating. Participants selected this study based on schedule availability, without knowledge of study content. The electronic informed consent procedure included several questions to confirm that participants understood their rights; participants provided informed consent electronically by agreeing to participate. Consistent with this study's focus on young adults, participants over the age of $25(n=14)$ were excluded from the analyses. The remaining 382 participants in the sample were mostly female $(60.2 \%)$ and college-aged $(M=19.51$, $S D=1.35)$, reflecting the demographics of the human subjects pool at the university. The ethnic identity distribution of this sample was $67.5 \%$ White or European American; $13.1 \%$ Asian; 2.4\% Native Hawaiian or Other Pacific Islander; 1.6\% Black or African American; .3\% American Indian or Alaska Native; $13.9 \%$ Multiethnic; and $1.3 \%$ unknown. The final sample was 362 because of missing data (see below).

\section{Measures}

Betrayal trauma was measured with the Brief Betrayal Trauma Survey (BBTS; Goldberg \& Freyd, 2006), a 28-item 


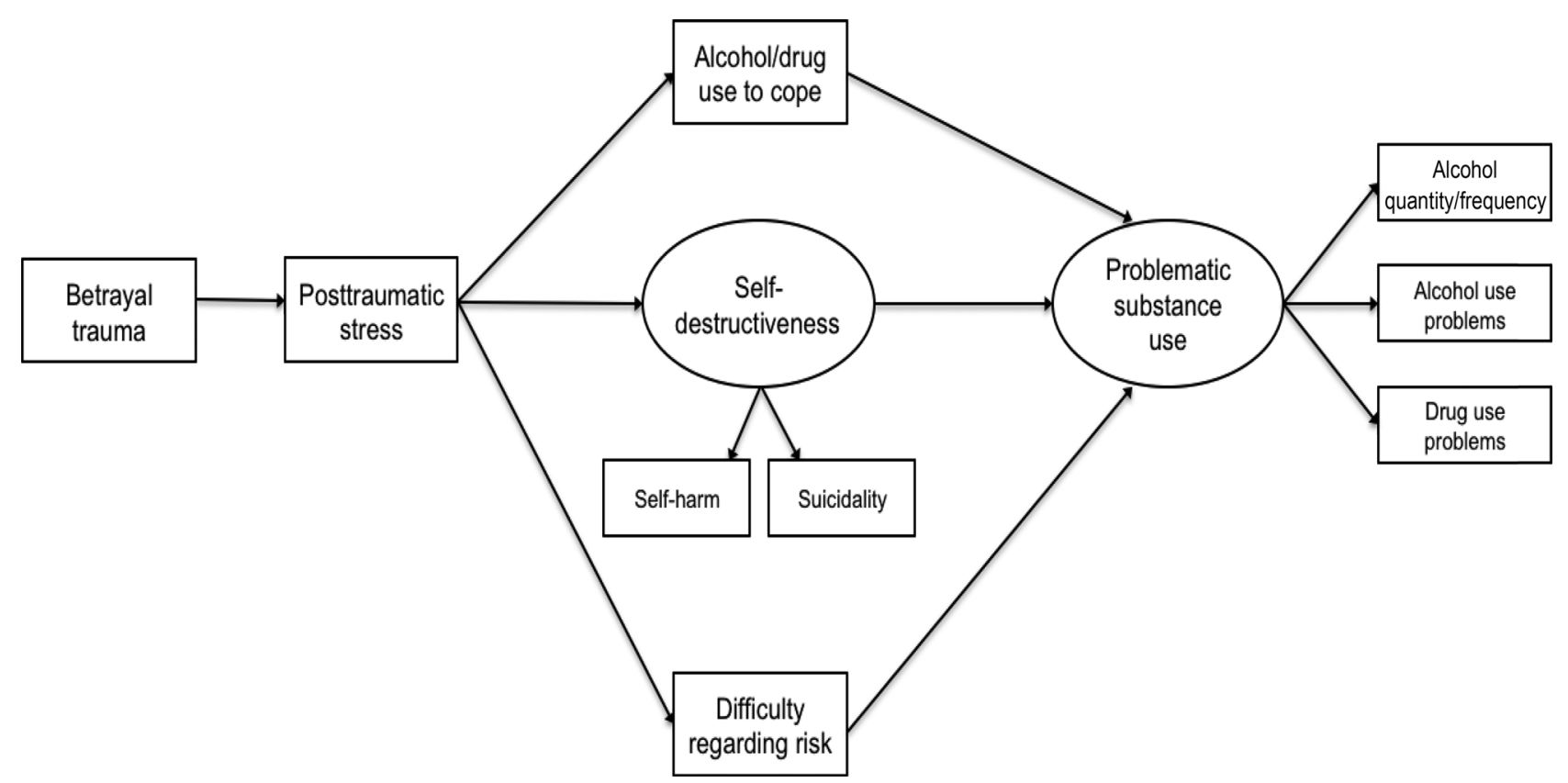

Figure 1. Original hypothesized structural equation model of pathways from higher betrayal trauma to problematic substance use. Residual variance components and direct effects of betrayal on self-destructiveness and on difficulty regarding risk are omitted for clarity of presentation.

self-report survey of potentially traumatic experiences that are low, medium, and high in betrayal, an index of the victim's relational closeness with the perpetrator. Participants indicate if each event happened to them before and after age 18 , and if so, how often: never $=0$, one or two times $=1$, more than that $=2$. Sample items include "You were made to have some form of sexual contact, such as touching or penetration, by someone with whom you were very close (such as a parent or lover)" (high betrayal); "You were made to have such sexual contact by someone with whom you were not close" (medium betrayal); and "You were in a major earthquake, fire, flood, hurricane, or tornado that resulted in significant loss of personal property, serious injury to yourself or a significant other, the death of a significant other, or the fear of your own death" (low betrayal). The BBTS has demonstrated good construct validity and test-retest reliability (Goldberg \& Freyd, 2006). In the present study, betrayals that met threshold for medium- or high-betrayal trauma were combined (mean scored) into a single index score of higher betrayal trauma, whereas betrayals that met threshold for low-betrayal trauma (e.g. natural disaster, car accident) were combined into a single index of lower betrayal trauma, a control variable in the analysis.

The PTSD Checklist for civilians (PCL-C; Ruggiero, Del Ben, Scotti, \& Rabalais, 2003) was used to assess past-year posttraumatic symptoms. The PCL-C contains five items that assess reexperiencing, seven items that assess avoidance and numbing, and five items that assess increased arousal. The PCL$\mathrm{C}$ demonstrated good internal consistency and test-retest reliability with a college undergraduate sample (Conybeare, Behar, Solomon, Newman, \& Borkovec, 2012). Coefficient $\alpha$ for the PCL-C in the present analytic sample was .95 .
Eight question items, developed by the authors, were used to assess difficulty regarding risk to self and others. The concept of risk was introduced in the following way: "Below is a list of potential risks that people sometimes experience. Please read each one carefully and select the response that indicates whether you have experienced that risk in the past year." Items assess difficulty discerning risk (e.g., "Been unable to decide whether a person or situation was safe?"), and difficulty heeding potential risk to self (e.g., "Behaved in ways that put your safety at risk?") or others (e.g., "Made choices that were risky for other people near you or for people dependent on you?"). To account for the possibility that some of those who take risks may not subjectively evaluate their behavior as risky, we included items that require normative judgment (e.g., "Made choices that other people might think are dangerous?"). Responses to each item were yes or no, yielding a checklist-type sum total score. Participants in the current study endorsed all eight risk items and a principal components factor analysis did not indicate multidimensionality (eigenvalue for first component $=4.00$, with $50.0 \%$ of variance explained). Coefficient $\alpha$ for the risk items in the present sample was .85 .

The Deliberate Self-Harm Inventory (DSHI; Gratz, 2001) was used to assess nonsuicidal self-injurious behavior. For each of the 17 DSHI index questions, participants answer yes or no, and if yes, how many times. Index and frequency questions are multiplied to yield a past-year self-harm quantity and frequency score. The DSHI has demonstrated good internal consistency and adequate construct, convergent, and discriminant validity (Gratz, 2001). In this sample, participants endorsed 14 of the 17 DSHI items and the coefficient $\alpha$ was .75 . 
Suicidality was assessed with three self-report items adapted from the Suicidal Behavior Questionnaire (Linehan, 1996). Participants responded on Likert-type scales to three questions about past-year thoughts of dying, suicidal ideation, and suicide attempt. These three items were combined with mean scoring into a single composite of suicidality. Coefficient $\alpha$ of the standardized items was .78. A principal components factor analysis did not indicate multidimensionality (eigenvalue for first component $=2.15$, with $71.7 \%$ of variance explained).

A modified version of the 13-item coping subscale of the Drinking Motives Questionnaire-Revised (DMQ-R; Cooper, 1994) was used to assess frequency of coping as a motivation for both alcohol and other drug use. Participants are instructed to "indicate how often you drink alcohol or use other drugs for each reason," on a 5-point scale from almost never/never to almost always/always. Previous researchers (e.g., Simons, Correia, Carey, \& Borsari, 1998) have modified the DMQ-R to probe motives for use of other drugs besides alcohol. Internal consistency of the DMQ-R in an undergraduate sample was excellent for coping motives for alcohol and marijuana use (Simons et al., 1998). Coefficient $\alpha$ for the DMQ-R in this sample was .95.

Participants self-reported on alcohol consumption quantity and frequency within a typical month in the past year. The 10-item Alcohol Use Disorders Identification Test (AUDIT; Babor, Higgins-Biddle, Saunders, \& Monteiro, 2001) was used to assess past-year alcohol use problems. The AUDIT measures hazardous consumption (e.g., quantity and frequency levels), dependence symptoms (e.g., impaired control over drinking), and harmful use (e.g., alcohol-related injuries). The AUDIT has good test-retest reliability, predictive validity, and convergent validity (Babor et al., 2001). In this study, we scored the AUDIT to produce a separate, 7-item assessment of harmful use and alcohol dependence only (Babor et al., 2001), to reduce overlap with the alcohol quantity and frequency index. Coefficient $\alpha$ of the 7-item AUDIT measure was .77.

Past-year drug use problems were assessed with the 11item Drug Use Disorders Identification Test (DUDIT; Berman, Bergman, Palmstierna, \& Schlyter, 2003). Components of problematic drug use are harmful use, loss of control, craving, and prioritization of drug use (Berman et al., 2003). The DUDIT has been administered online with general populations aged 15 and above as a self-report screening instrument for drugrelated problems and has obtained excellent internal consistency (Sinadinovic, Berman, Hasson, \& Wennberg, 2010). In this study, internal consistency was excellent $(\alpha=.89)$.

\section{Data Analysis}

Data screening and descriptive analysis (see Table 1) were conducted prior to hypothesis testing. The two quantity and frequency indexes (alcohol use and self-harm) were logtransformed prior to analyses. Inspection of the data for missingness indicated that 16 participants did not complete the Brief Betrayal Trauma Survey (BBTS) regarding experiences prior to age 18 , whereas four participants completed only the BBTS. Follow-up $t$ test indicated that participants who did not complete the BBTS had significantly higher posttraumatic stress symptom scores on the PCL-C than participants who completed the BBTS, $t(374)=4.14, p<.001$. We excluded participants with missing BBTS data from the analysis. In addition, we excluded the participants who completed only the BBTS. The final analytic sample consisted of 362 participants.

A structural equation model estimating pathways from higher betrayal trauma to problematic substance use was analyzed using Mplus Version 6.12 (Muthén \& Muthén, 1998-2010), with maximum likelihood estimation. Direct paths from betrayal to self-destructiveness and to difficulty regarding risk were included, to reflect the theoretically driven assumption that posttraumatic stress is not a necessary condition for these experiences. Disturbance terms for the intervening variables and for the indicators of problematic substance use were included to account for possible common omitted causes. The second hypothesis was analyzed with the addition of lowerbetrayal trauma in the analysis. The significance of indirect effects was tested using bootstrapping (Hayes \& Scharkow, 2013). Confidence intervals reported are bias-corrected bootstrap confidence intervals for the standardized indirect effects.

Structural model fit to the observed data was evaluated with global fit indexes (Kline, 2011). To address areas of poor model fit, we inspected model modification indices and respecified several model parameters on the basis of empirical and theoretical criteria (Sörbom, 1989). To prevent capitalizing on chance in the respecification process, we randomly divided the sample in half and estimated the hypothesized model on one half of the sample $(n=184)$, then estimated the respecified model on the second half $(n=178)$. The two randomly divided halves of the sample did not differ significantly on any demographic or study variables.

\section{Results}

Means, standard deviations, and correlations among the primary study variables are presented in Table 1 . Almost half of the study sample $(45.0 \%)$ reported experiencing one or more higher betrayal traumas prior to age 18 . The most common higher betrayal traumas in the sample were being emotionally or psychologically mistreated over a significant period by someone very close $(25.4 \%)$ or someone not close $(15.5 \%)$, along with witnessing physical or sexual assault, emotional mistreatment, or neglect occur to someone very close (24.3\%). In addition, $12.4 \%$ of the sample was neglected by someone very close; $9.7 \%$ was made to have sexual contact by someone very close; and $5.3 \%$ was physically assaulted by someone very close. Participants endorsed all nine higher betrayal trauma items.

The majority of the sample (83.2\%) reported consuming alcohol in the past year. Weekly binge drinking, defined as consuming four or more drinks on one occasion, was reported 
Table 1

Means, Standard Deviations, and Intercorrelations Among Variables in Structural Model

\begin{tabular}{|c|c|c|c|c|c|c|c|c|c|c|}
\hline & $M$ & $S D$ & 1 & 2 & 3 & 4 & 5 & 6 & 7 & 8 \\
\hline 1. BT & .83 & 1.32 & & & & & & & & \\
\hline 2. PCL & 33.63 & 13.29 & $.49 * * *$ & & & & & & & \\
\hline 3. DMQ & 21.47 & 10.64 & $.23 * * *$ & $.33 * * *$ & & & & & & \\
\hline 4. DSH & .31 & .89 & $.27 * * *$ & $.39 * * *$ & $.20 * * *$ & & & & & \\
\hline 5. SUI & .75 & .99 & $.31 * * *$ & $.47 * * *$ & $.32 * * *$ & $.39 * * *$ & & & & \\
\hline 6. RISK & 2.75 & 2.63 & $.19 * * *$ & $.34 * * *$ & $.42 * * *$ & $.18^{* *}$ & $.27 * * *$ & & & \\
\hline 7. ALC & 1.98 & 1.30 & -.08 & -.05 & $.27 * * *$ & -.04 & .00 & $.26 * * *$ & & \\
\hline 8. AUD & .33 & .52 & $.20 * * *$ & $.21 * * *$ & $.38^{* * *}$ & $.12^{*}$ & $.20 * * *$ & $.39 * * *$ & $.41 * * *$ & \\
\hline 9. DUD & 3.33 & 5.76 & $.23 * * *$ & $.26 * * *$ & $.50 * * *$ & $.13^{*}$ & $.30 * * *$ & $.38 * * *$ & $.31 * * *$ & $.32 * * *$ \\
\hline
\end{tabular}

Note. $N=362 . \mathrm{BT}=$ experiences of higher betrayal trauma prior to age 18 , mean-scored; PCL $=$ PTSD Checklist-Civilians Version; DMQ $=$ Drinking Motives Questionnaire-Coping subscale (adapted to assess coping motives for alcohol and other drug use); DSH = Deliberate Self-Harm Inventory quantity and frequency; SUI = suicidality; RISK = difficulty regarding risk; ALC = alcohol quantity and frequency; AUD = Alcohol Use Disorders Identification Test-Harmful Alcohol Use and Alcohol Dependence subscales; DUD = Drug Use Disorders Identification Test.

$* p<.05 . * * p<.01 . * * * p<.001$

by almost one quarter of the sample (23.2\%). According to recommended AUDIT cutoff scores, $38.4 \%$ of participants met criteria for hazardous and harmful alcohol use. Use of drugs besides alcohol was reported by $44.5 \%$ of the sample, with a modal frequency of once a month or less often. Among participants who disclosed using illicit drugs in the past year, $19.9 \%$ reported using drugs 2-3 times per week and $21.3 \%$ reported using drugs 4 or more times per week. According to recommended DUDIT cutoffs (calibrated differently for women and men), $30.7 \%$ of the sample met clinical criteria for drug-related problems.

First, we evaluated the hypothesized pathways from higherbetrayal trauma to problematic substance use. Using half of the sample, we examined (a) the direct effects of higher betrayal trauma prior to age 18 on posttraumatic stress symptoms; (b) the direct effect of posttraumatic stress symptoms on substance use to cope with negative affect, self-destructiveness, and difficulty regarding risk; and (c) the direct effects of these three intervening variables on problematic substance use. Based on global fit indices, the overall structural model presented in Figure 1 demonstrated inadequate fit to the data, $\chi^{2}(18)=65.61$, $p<.001$; comparative fit index $(\mathrm{CFI})=.85$; root mean square error of approximation (RMSEA) $=.12$. All observed indicators significantly loaded onto latent constructs, indicating adequacy of the measurement models of self-destructiveness and problematic substance use. In addition, all direct paths specified in Figure 1 were significant, with the exception of the paths from betrayal to self-destructiveness and from self-destructiveness to problematic substance use.

The modification index suggested that model fit would improve if substance use to cope with negative affect were an indicator of the problematic substance use latent variable (modification index $=16.4$, expected parameter change $=13.8$ ), rather than an intervening pathway between betrayal trauma and problematic substance use. In support of this modification, the alcohol quantity and frequency index's standardized factor loading on the problematic substance use latent variable (.43) was significant, but the estimate was approximately half the magnitude of the loading of drug use problems (.76), suggesting that the convergent validity of the alcohol quantity and frequency index was questionable. In a young adult, college sample, higher alcohol consumption may be normative (Griffin, 2010), making quantity and frequency a less valid indicator of problematic substance use in this particular sample.

Using the second half of the sample, we analyzed a respecified model in which problematic substance use was indexed by alcohol use problems, drug use problems, and substance use to cope with negative affect (see Figure 2). The respecified model demonstrated excellent fit to the data from the second half of the sample, $\chi^{2}(14)=16.51, n s ; \mathrm{CFI}=.99$; RMSEA $=.03$. Thus, we combined the sample halves to inspect model fit statistics and path coefficients in the full sample $(n=362), \chi^{2}(14)=15.96, n s ; \mathrm{CFI}=1.00 ; \mathrm{RMSEA}=.02$. Higher betrayal trauma prior to age 18 was associated with greater symptoms of posttraumatic stress, which in turn was associated with greater self-destructiveness and difficulty regarding risk. Self-destructiveness and difficulty regarding risk were associated with greater problematic substance use. All observed indicators loaded significantly onto the problematic substance use construct. Overall, 50.9\% of the variance in problematic substance use was explained by the model.

Next, we analyzed the indirect effect of higher betrayal trauma experiences prior to age 18 on problematic substance use. We found a significant total indirect effect of higher betrayal trauma on problematic substance use, $\beta=.28$, $p<.001,95 \%$ CI $[.15, .41]$. Both specific indirect effect pathways from betrayal to problematic substance use via posttraumatic stress were significant: the pathway from posttraumatic stress symptoms to self-destructiveness, $95 \% \mathrm{CI}[.06, .18]$, and from posttraumatic stress symptoms to difficulty regarding risk, 


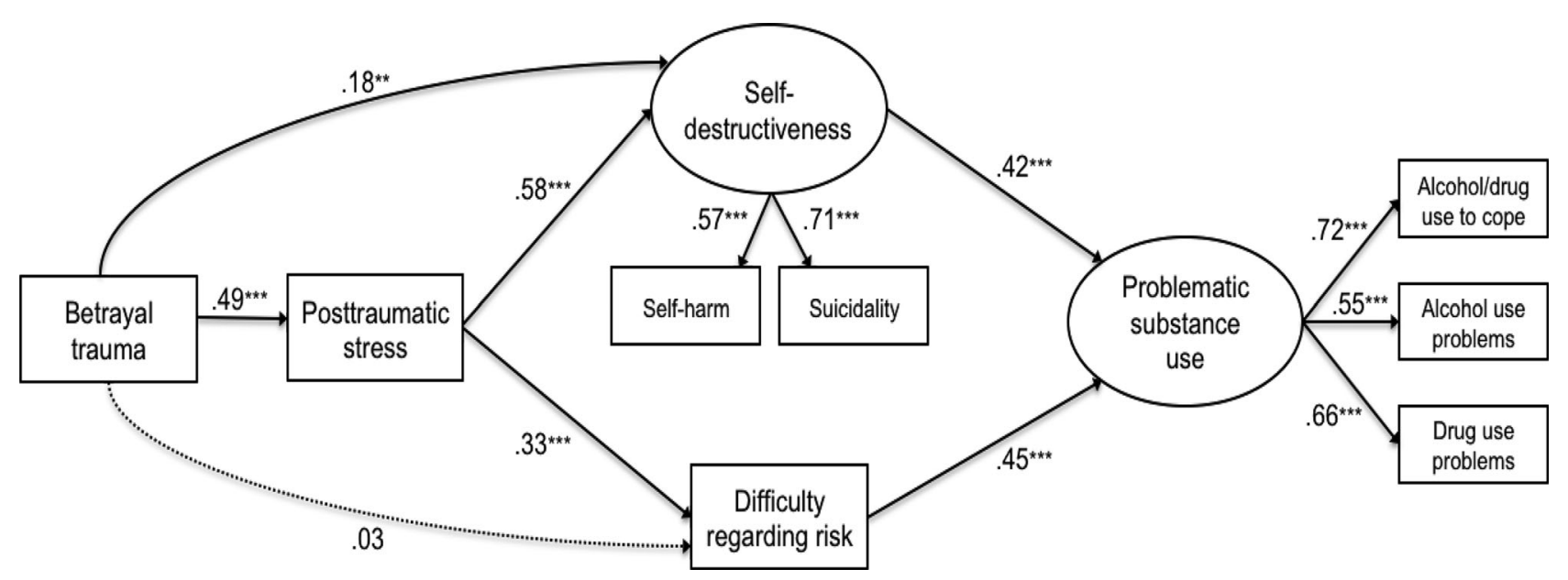

Figure 2. Final structural equation model of pathways from higher betrayal trauma to problematic substance use. Coefficients are standardized estimates; dotted path is $n s$. Residual variance components are omitted for clarity of presentation. $* * p<.01 . * * * p<.001$.

95\% CI [.03, .11]. The specific indirect effects of higher betrayal trauma on problematic substance use via self-destructiveness only and via difficulty regarding risk only, without posttraumatic stress, were not significant.

To evaluate the prediction that higher betrayal trauma would be associated with problematic substance use while controlling for lower betrayal trauma, we tested a competing bootstrap model of indirect effects that included lower betrayal trauma prior to age 18 (reported by $35.6 \%$ of the sample). Lower betrayal trauma was not a significant predictor of posttraumatic stress symptoms $(\beta=.07, n s)$, nor did lower betrayal trauma have a significant indirect effect on problematic substance use, 95\% CI [-.02, .08]. Furthermore, the inclusion of lower betrayal trauma in the model resulted in a significant decrease in overall model fit, with $\chi^{2}(21)=46.00$, $p=.001$, whereas all higher betrayal paths remained significant. Therefore, it appears that the experience of lowerbetrayal trauma does not account for the relations presented in Figure 2.

\section{Discussion}

This study examined the relation between betrayal trauma experienced prior to age 18 and problematic substance use, evaluating two novel intervening pathways (self-destructiveness and difficulty regarding risk) and a pathway proposed in recent research (substance use to cope with negative affect). Optimal fit to the data was obtained when we respecified the model to make coping an indicator of problematic substance use, rather than an intervening pathway to problematic use. In the final model (see Figure 2), higher betrayal trauma was associated with problematic substance use via posttraumatic stress and two intervening pathways: self-destructiveness and difficulty regarding risk. Lower betrayal trauma prior to age 18 was not associated with problematic substance use, nor was lower betrayal trauma associated with past-year posttraumatic stress. These findings reinforce prior research on childhood cumulative trauma and on betrayal trauma theory that has found more complex, negative psychosocial outcomes in survivors of interpersonal trauma, as compared to noninterpersonal trauma (Briere, Kaltman, \& Green, 2008; Cloitre et al., 2009; Goldsmith, Chesney, Heath, \& Barlow, 2013). In addition, the finding that higher betrayal trauma was associated with problematic substance use only when posttraumatic stress was included in each indirect pathway suggests that betrayal-exposed individuals who exhibit self-destructiveness and difficulty regarding risk may not engage in problematic substance use unless there is co-occurring posttraumatic stress.

If substance use represents a form of coping or selfmedication (Khantzian, 1997) for some interpersonal trauma survivors suffering from posttraumatic stress, a troubling question remains. Why do those who have been harmed by others choose to cope with distress in ways that may harm themselves? Our findings extend prior research by contributing evidence in support of a novel conceptual model of how betrayal trauma relates to problematic substance use. This model suggests that betrayal-exposed individuals who use substances may not recognize their risk for harm. Additionally, they may use substances as a more or less intentional means of self-destruction. Each of these proposed pathways suggests compelling directions for future research.

Longitudinal research on the cognitive, behavioral, and psychobiological components of risk taking among betrayalexposed youth will increase understanding of when and how difficulties regarding risk emerge. To extend this study's global assessment of risk-evaluation difficulties, future research should evaluate risky decisions made specifically within the context of substance use. An experimental paradigm that requires evaluating risk, followed by a decision-making task relevant to substance use (Kreek, Nielsen, Butelman, \& LaForge, 2005), could provide insight into important questions such as whether and under what conditions survivors discern risk but disregard 
it, or simply do not discern the risk. A deeper understanding of the risk-evaluation process among those exposed to betrayal has the potential to inform prevention strategies that help these adolescents and young adults make healthy decisions in the context of substance use, sexual activity, and other potentially risky domains.

Further research can clarify the extent to which betrayal survivors use psychoactive substances self-destructively, to access a potentially lethal continuum from loss of consciousness to death (Rothman et al., 2010). In addition to extending the evidence base on self-destructiveness as a motive for substance use, future research can evaluate perceived life threat (Kelley, Weathers, Mason, \& Pruneau, 2012) as a dimension of trauma that may increase the likelihood of self-destructiveness. Of course, an alternative explanation to our findings on betrayal and self-destructiveness is that self-destructiveness, like substance use, is a means of coping with negative affect. Indeed, tension reduction is one of the most common selfreported reasons for self-harming behavior (Klonsky, 2007). It is noteworthy, however, that the best-fitting model to our data was a model that distinguished self-destructiveness from substance use, rather than a model that subsumed self-harm, suicidality, and substance use under a single "tension reduction" construct (alternative model results available from the authors upon request). This provides intriguing evidence that for some survivors of betrayal or under certain conditions, the functions of self-harm and suicidality may be distinct from tension reduction.

Interpretation of study findings should be considered in light of at least three limitations. First, the model we estimated with cross-sectional data describes theoretically driven correlations among study variables, relations that are not definitively causal. Further research, especially longitudinal designs, will be needed to support the direction of effects described in this study. Second, use of an undergraduate sample limits the generalizability of our findings, though it should be emphasized that consistent with prior research with college students (Read et al., 2012), both trauma exposure and problematic substance use were prevalent in our sample, and participants did not selfselect into our study. Third, self-report measurement relies on participant willingness to disclose personal experiences and to recall and report past events accurately. Study findings were obtained after excluding 16 participants who did not self-report on their experiences of early-life betrayal. Although these 16 participants were not asked to explain why they omitted BBTS responses, it is possible that they deemed their personal experiences too private to disclose or too distressing to ponder for the purpose of the questionnaire. Consistent with betrayal trauma theory, awareness or memory of betrayal may have been attenuated for some of these participants. As a result, findings may underestimate the true frequency of betrayal in the sample (Hardt \& Rutter, 2004), and may not generalize to all individuals with a history of betrayal. In research underway, we are investigating alternative methods to assess betrayal history, including measures of implicit associations between the self and betrayal (Lindgren, Kaysen, Werntz, Gasser, \& Teachman, 2013).

This study highlights the enduring health- and safety-risking outcomes associated with trauma that involves social betrayal, as compared to trauma low in betrayal. Our findings suggest that even populations not actively seeking treatment for trauma-related symptoms may exhibit elevated rates of potentially harmful substance use behaviors that are indirectly associated with their early-life trauma histories. Research and clinical inquiry can benefit from further studies aimed at understanding how to support betrayal trauma survivors whose psychosocial vulnerabilities, such as difficulty discerning and heeding risk, may put them at elevated risk for problematic substance use.

\section{References}

Afifi, T. O., Henriksen, C. A., Asmundson, G. J. G., \& Sareen, J. (2012). Childhood maltreatment and substance use disorders among men and women in a nationally representative sample. Canadian Journal of Psychiatry, 57, $677-685$.

Babor, T. F., Higgins-Biddle, J. C., Saunders, J. B., \& Monteiro, M. G. (2001). The alcohol use disorders identification test: Guidelines for use in primary care (2nd ed.). Geneva, Switzerland: World Health Organization Department of Mental Health and Substance Dependence.

Berman, A. H., Bergman, H., Palmstierna, T., \& Schlyter, F. (2003). DUDIT: The Drug Use Disorders Identification Test manual. Stockholm, Sweden: Karolinska Institutet.

Bornovalova, M. A., Tull, M. T., Gratz, K. L., Levy, R., \& Lejuez, C. W. (2011). Extending models of deliberate self-harm and suicide attempts to substance users: Exploring the roles of childhood abuse, posttraumatic stress, and difficulties controlling impulsive behavior when distressed. Psychological Trauma: Theory, Research, Practice, and Policy, 3, 349-359. doi: $10.1037 / \mathrm{a} 0021579$

Brady, K. T., Back, S. B., \& Coffey, S. F. (2004). Substance abuse and posttraumatic stress disorder. Current Directions in Psychological Science, 13, 206-209. doi:10.1111/j.0963-7214.2004.00309.x

Briere, J., Kaltman, S., \& Green, B. L. (2008). Accumulated childhood trauma and symptom complexity. Journal of Traumatic Stress, 21, 223-226. doi: $10.1002 /$ jts. 20317

Briere, J., Hodges, M., \& Godbout, N. (2010). Traumatic stress, affect dysregulation, and dysfunctional avoidance: A structural equation model. Journal of Traumatic Stress, 23, 767-274. doi:10.1002/jts.20578

Budden, A. (2009). The role of shame in posttraumatic stress disorder: A proposal for a socio-emotional model for DSM-V. Social Science \& Medicine, 69, 1032-1039. doi:10.1016/j.socscimed.2009.07.032

Chilcoat, H. D., \& Menard, C. (2003). Epidemiological investigations: Comorbidity of posttraumatic stress disorder and substance use disorder. In P. Ouimette \& P. J. Brown (Eds.), Trauma and substance abuse (pp. 9-28). Washington, DC: American Psychological Association.

Cloitre, M., Stolbach, B. C., Herman, J. L., van der Kolk, B., Pynoos, R., Wang, J., \& Petkova, E. (2009). A developmental approach to complex PTSD: Childhood and adult cumulative trauma as predictors of symptom complexity. Journal of Traumatic Stress, 22, 399-408. doi:10.1002/jts. 20444

Conybeare, D., Behar, E., Solomon, A., Newman, M. G., \& Borkovec, T. D. (2012). The PTSD Checklist-Civilian Version: Reliability, validity, and 
factor structure in a nonclinical sample. Journal of Clinical Psychology, 68, 699-713. doi:10.1002/jclp.21845

Cooper, M. L. (1994). Motivations for alcohol use among adolescents: Development and validation of a four-factor model. Psychological Assessment, 6, 117-128. doi:10.1037/1040-3590.6.2.117

DePrince, A. P. (2005). Social cognition and revictimization risk. Journal of Trauma \& Dissociation, 6, 125-141. doi:10.1300/J229v06n01_08

Duncan, A. E., Sartor, C. E., Scherrer, J. F., Grant, J. D., Heath, A. C., Nelson, E. C., ... Bucholz, K. K. (2008). The association between cannabis abuse and dependence and childhood physical and sexual abuse: Evidence from an offspring of twins design. Addiction, 103, 990-997. doi:10.1111/j.13600443.2008.02210.x

Edwards, V. J., Freyd, J. J., Dube, S. R., Anda, R. F., \& Felitti, V. J. (2012). Health outcomes by closeness of sexual abuse perpetrator: A test of betrayal trauma theory. Journal of Aggression, Maltreatment \& Trauma, 21, 133148. doi:10.1080/10926771.2012.648100

Freyd, J. J., DePrince, A. P., \& Gleaves, D. H. (2007). The state of betrayal trauma theory: Reply to McNally. Memory, 15, 295-311. doi:10.1080/09658210701256514

Glantz, M. D. (2010). Touchstone issues for theories of substance abusedependence etiology. In L. M. Scheier (Ed.), Handbook of drug use etiology: Theory, methods, and empirical findings. Washington, DC: American Psychological Association.

Goldberg, L. R., \& Freyd, J. J. (2006). Self-reports of potentially traumatic experiences in an adult community sample: Gender differences and testretest stabilities of the items in a brief betrayal-trauma survey. Journal of Trauma \& Dissociation, 7, 39-63. doi:10.1300/J229v07n03_04

Goldsmith, R. E., Chesney, S. A., Heath, N. M., \& Barlow, M. R. (2013). Emotion regulation difficulties mediate associations between betrayal trauma and symptoms of posttraumatic stress, depression, and anxiety. Journal of Traumatic Stress, 26, 376-384. doi:10.1002/jts.21819

Gratz, K. L. (2001). Measurement of deliberate self-harm: Preliminary data on the deliberate self-harm inventory. Journal of Psychopathology and Behavioral Assessment, 23, 253-263. doi:10.1023/A:1012779403943

Greeley, J., \& Oei, T. (1999). Alcohol and tension reduction. In K. E. Leonard \& H. T. Blane (Eds.), Psychological theories of drinking and alcoholism (2nd ed., pp. 14-53). New York, NY: Guilford Press.

Griffin, K. W. (2010). The epidemiology of substance use among adolescents and young adults: A developmental perspective. In L. M. Scheier (Ed.), Handbook of drug use etiology: Theory, methods, and empirical findings (pp. 73-92). Washington, DC: American Psychological Association.

Hardt, J., \& Rutter, M. (2004). Validity of adult retrospective reports of adverse childhood experiences: Review of the evidence. Journal of Child Psychology and Psychiatry, 45, 260-273. doi:10.1111/j.1469-7610.2004.00218.x.

Hayes, A. F., \& Scharkow, M. (2013). The relative trustworthiness of inferential tests of the indirect effect in statistical mediation analysis: Does method really matter? Psychological Science, 24, 1918-1927. doi: $10.1177 / 0956797613480187$

Herman, J. L. (1997). Trauma and recovery: The aftermath of violence from domestic abuse to political terror. New York, NY: Basic Books.

Kaysen, D., Dillworth, T. M., Simpson, T., Waldrop, A., Larimer, M. E., \& Resick, P. A. (2007). Domestic violence and alcohol use: Trauma-related symptoms and motives for drinking. Addictive Behaviors, 32, 1272-1283. doi:10.1016/j.addbeh.2006.09.007

Kelley, L. P., Weathers, F. W., Mason, E. A., \& Pruneau, G. M. (2012). Association of life threat and betrayal with posttraumatic stress disorder symptom severity. Journal of Traumatic Stress, 25, 408-415. doi:10.1002/jts.21727
Khantzian, E. J. (1997). The self-medication hypothesis of substance use disorders: A reconsideration and recent applications. Harvard Review of Psychiatry, 4, 231-234. doi:10.3109/10673229709030550

Kline, R. B. (2011). Principles and practice of structural equation modeling (3rd ed.). New York, NY: Guilford Press.

Klonsky, E. D. (2007). The functions of deliberate self-injury: A review of the evidence. Clinical Psychology Review, 27, 226-239. doi:10.1016/j.cpr.2006.08.002

Kreek, M. J., Nielsen, D. A., Butelman, E. R., \& LaForge, K. S. (2005). Genetic influences on impulsivity, risk taking, stress responsivity and vulnerability to drug abuse and addiction. Nature Neuroscience, 8, 1450-1457. doi: $10.1038 / \mathrm{nn} 1583$

Lindgren, K. P., Kaysen, D., Werntz, A. J., Gasser, M. L., \& Teachman, B. A. (2013). Wounds that can't be seen: Implicit trauma associations predict posttraumatic stress disorder symptoms. Journal of Behavior Therapy and Experimental Psychiatry, 44, 368-375. doi:10.1016/j.jbtep.2013.03.003

Linehan, M. M. (1996). The Suicidal Behaviors Questionnaire-14 (SBQ-14). Unpublished instrument, University of Washington, Seattle, WA.

Messman-Moore, T. L., \& Brown, A. L. (2006). Risk perception, rape, and sexual revictimization: A prospective study of college women. Psychology of Women Quarterly, 30, 159-172. doi:10.1111/j.1471-6402.2006.00279.x

Muthén, L. K., \& Muthén, B. O. (1998-2010). Mplus user's guide (6th ed.). Los Angeles, CA: Author.

Panagioti, M., Gooding, P. A., \& Tarrier, N. (2012). A meta-analysis of the association between posttraumatic stress disorder and suicidality: The role of comorbid depression. Comprehensive Psychiatry, 53, 915-930. doi:10.1016/j.comppsych.2012.02.009

Peters, E. N., Khondkaryan, E., \& Sullivan, T. P. (2012). Associations between expectancies of alcohol and drug use, severity of partner violence, and posttraumatic stress among women. Journal of Interpersonal Violence, 27, 2108-2127. doi:10.1177/0886260511432151

Platt, M., \& Freyd, J. (2012). Trauma and negative underlying assumptions in feelings of shame: An exploratory study. Psychological Trauma: Theory, Research, Practice, and Policy, 4, 370-378. doi:10.1037/a0024253

Read, J. P., Colder, C. R., Merrill, J. E., Ouimette, P., White, J., \& Swartout, A. (2012). Trauma and posttraumatic stress symptoms predict alcohol and other drug consequence trajectories in the first year of college. Journal of Consulting and Clinical Psychology, 80, 426-439. doi:10.1037/a0028210

Rothman, E. F., Bernstein, J., \& Strunin, L. (2010). Why might adverse childhood experiences lead to underage drinking among US youth? Substance Use \& Misuse, 45, 2281-2290. doi:10.3109/10826084.2010.482369

Ruggiero, K. J., Del Ben, K., Scotti, J. R., \& Rabalais, A. E. (2003). Psychometric properties of the PTSD Checklist-Civilian Version. Journal of Traumatic Stress, 16, 495-502. doi:10.1023/A:1025714729117

Simons, J., Correia, C. J., Carey, K. B., \& Borsari, B. E. (1998). Validating a five-factor marijuana motives measure: Relations with use, problems, and alcohol motives. Journal of Counseling Psychology, 45, 265-273. doi:10.1037/0022-0167.45.3.265

Sinadinovic, K., Berman, A. H., Hasson, D., \& Wennberg, P. (2010). Internetbased assessment and self-monitoring of problematic alcohol and drug use. Addictive Behaviors, 35, 464-470. doi:10.1016/j.addbeh.2009.12.021

Sörbom, D. (1989). Model modification. Psychometrica, 54, 371-384. doi:10.1007/BF02294623

Staiger, P. K., Melville, F., Hides, L., Kambouropoulos, N., \& Lubman, D. I. (2009). Can emotion-focused coping help explain the link between posttraumatic stress disorder severity and triggers for substance use 
in young adults? Journal of Substance Abuse Treatment, 36, 220-226. doi:10.1016/j.jsat.2008.05.008

Strom, T. Q., Leskela, J., James, L. M., Thuras, P. D., Voller, E., Weigel, R., Holz, K. B. (2012). An exploratory examination of risk-taking behavior and PTSD symptom severity in a veteran sample. Military Medicine, 177, 390-396.

Substance Abuse and Mental Health Services Administration. (2012). Results from the 2010 National Survey on Drug Use and Health: Mental health findings, NSDUH Series H-42, HHS Publication No. (SMA) 11-4667. Rockville, MD: Author.
Ullman, S. E., Filipas, H. H., Townsend, S. M., \& Starzynski, L. L. (2007). Psychosocial correlates of PTSD symptom severity in sexual assault survivors. Journal of Traumatic Stress, 20, 821-831. doi:10.1002/jts.20290

Waldrop, A. E., Back, S. E., Verduin, M. L., \& Brady, K. T. (2007). Triggers for cocaine and alcohol use in the presence and absence of posttraumatic stress disorder. Addictive Behaviors, 32, 634-639. doi:10.1016/j.addbeh.2006.06.00

Weierich, M. R., \& Nock, M. K. (2008). Posttraumatic stress symptoms mediate the relation between childhood sexual abuse and nonsuicidal self-injury. Journal of Consulting and Clinical Psychology, 76, 39-44. doi:10.1037/0022-006X.76.1.39 